\section{The reality of} undergraduate GP teaching: a medical student's perspective

We read with great interest the paper written by Cottrell and colleagues, which attempted to quantify the average time allocated to GP teaching across UK medical schools. ' We acknowledge the importance of identifying strategies to increase interest in general practice and would like to highlight the importance of a positive student experience in achieving this goal.

When quantifying total GP teaching time, this study did not differentiate between lectures and clinical experience, based on the assumption that it is the quantity of GP teaching which correlates to an increased percentage of GP graduates. Although this assumption is supported by other data within this field, it is important to note that evidence has shown that this correlation is only statistically significant for clinical-based teaching that allows patient contact. ${ }^{2}$ This correlation is not observed when accounting for total GP teaching time, including lectures and small-group tutorial work. Further data collection that makes the distinction between clinical and non-clinical experience would not only assess the quantity of effective GP teaching but also ensure that medical schools are appropriately informed about the type of improvements required to enhance interest in general practice.

Additionally, although this paper recognises that students' perceptions are further influenced by their overall experience, this is not reflected within the study method. Evidence from a recent paper assessing student experience in surgery emphasised the importance of a welcome environment, active educators, and opportunities for involvement when creating a positive experience with the specialty. ${ }^{3}$

An improved understanding of the features defining a positive clinical experience is particularly important given the difficulties of teaching during the COVID-19 pandemic. Student involvement is essential to ensure that GP practices continue to provide great learning opportunities. Given that telephone consultations have now become the norm, adapting to maintain student involvement will be yet another challenge for the GP.

Kaya-Maria S Kordelas,

Medical Student, University College London.

Email: kaya-maria.kordelas.15@aucl.ac.uk

Eva C Attubato,

Medical Student, University College London.

\section{REFERENCES}

1. Cottrell E, Alberti H, Rosenthal J, et al. Revealing the reality of undergraduate GP teaching in UK medical curricula: a cross-sectional questionnaire study. Br J Gen Pract 2020; DOI: https://doi. org/10.3399/bjgp20X712325.

2. Alberti H, Randles HL, Harding A, McKinley RK. Exposure of undergraduates to authentic GP teaching and subsequent entry to GP training a quantitative study of UK medical schools. $\mathrm{Br}$ J Gen Pract 2017; DOI: https://doi.org/10.3399/ bjgp17X689881.

3. Marshall DC, Salciccioli JD, Walton SJ, et al. Medical student experience in surgery influences their career choices: a systematic review of the literature. Surg Educ 2015; 72(3): 438-445.

DOI: https://doi.org/10.3399/bjgp20X713189

\section{Interdental and GP relationships in oral} \section{cancer}

We read your article titled 'Building bridges with dentistry' with great interest. 'It is vitally important for the academic community to continue to study oral health, so we thank you for your valuable contribution.

Oral cancer, a disease entity that is growing in prevalence in the UK, for which outcome is related to the timeliness of diagnosis, has been the focus of a growing body of research. Certainly, the development of a primary care-dentistry network, in principle, may improve the diagnostic process. However, as alluded to within your review, there are a range of factors that make procurement of a functioning network challenging

First, the engagement of the adult population with dental services in the UK is extremely poor. Unfortunately, the patient groups at greater risk of oral cancer lincreasing age, low socioeconomic background, male gender) tend to be the groups less likely to attend dental services. Our systematic review did not find any evidence to suggest that GPs are inferior to primary care dentists in the detection of oral cancer. ${ }^{2}$ We speculate that this may be due to the small number of cases diagnosed by primary care practitioners through their careers. While educational programmes within primary care are important, there should also be recognition that the disease itself is extremely variable. In our experiences, there have been patients presenting with symptoms outside the NICE checklist, who went on to receive a cancer diagnosis. These included: odynophagia, sore throat, weight loss, anorexia, and cervical dysphagia.

Second, an issue our research group expands upon in an upcoming publication (accepted BDJ, not yet available), for which we evaluate the diagnostic journeys patients with oral cancer took from primary care to secondary care, much of the diagnostic delay' took place within secondary care from interdisciplinary referrals (gastroenterology, maxillofacial surgery, ENT).

Unfortunately, the lack of current robust referral pathways between primary medical and dental services raises questions about the appropriateness for interprofessional referrals, as these would need to be auditable to assess prevention of delay in cancer diagnosis.

Jane Wilcock,

GP, Silverdale Medical Practice, Salford. Email: jane.wilcockanhs.net

Ciaran Grafton-Clarke,

Clinical Education Fellow, Leicester Royal Infirmary.

\section{REFERENCES}

1. Ahern J, Simon L, Barrow J Silk H. Building bridges with dentistry: NICE guideline supports collaborative practice between GPs and dentists. Br J Gen Pract 2020; DOI: 10.3399/bjgp20X712529.

2. Grafton-Clarke C, Chen KW, Wilcock J. Diagnosis and referral delays in primary care for oral squamous cell cancer: a systematic review. $\mathrm{Br}$ J Gen Pract 2019; DOI: https://doi.org/10.3399/ bjgp18X700205.

DOI: https://doi.org/10.3399/bjgp20X713201 